\title{
Funçóes de Base: O Ajuste Variacional
}

\author{
Nelson Henrique Morgon \\ Rogério Custódio* \\ roger@iqm.unicamp.br \\ Universidade Estadual de Campinas, Instituto de Química
}

\section{Palavras-Chaves}

Funções de base Funções hidrogenóides

Funções de Slater

Funções Gaussianas

Teorema de Hellmann-Feynman

\section{Resumo}

Dentre os modelos matemáticos utilizados para representar a estrutura eletrônica de sistemas microscópicos um dos mais valiosos é o modelo de Hartree-Fock [1]. O maior problema a ser resolvido neste modelo está na escolha das funções matemáticas a serem utilizadas para representar os orbitais de Hartree-Fock. Uma das sugestões mais importantes ao método Hartree-Fock foi formalizada por J.J.Roothaan através da técnica que ficou popularizada como o método da combinação linear de orbitais atômicos ou funções de base [2]. Em outras palavras, orbitais atômicos e moleculares podem ser obtidos como combinação linear de funções de base. Embora o modelo de Hartree-Fock-Roothaan tenha se tornado computacionalmente atrativo, para ser utilizado adequadamente impõem a solução de questões de caráter técnico, tais como:

- quais funções matemáticas podem ser utilizadas como conjuntos de base? e

- quantas funções de base devem ser utilizadas para representar adequadamente o sistema em estudo?

Os aspectos relacionados com as alternativas para responder a essas duas questões serão detalhados a seguir.

\section{Introdução}

As tentativas para obter uma solução para estas questões têm sido objeto de diversas revisões na literatura [3]. Em geral, estas revisões procuram organizar as informações disponíveis sobre o assunto e apresentar sugestões de escolha dos "melhores" conjuntos destas funções (ou conjuntos de base).

Uma análise dos testes e resultados apresentados na literatura, bem como o maior ou menor uso de determinadas funções de base, mostram que normalmente três critérios são considerados para nortear a escolha com relação ao tipo e número de funções de base:

- As funções de base devem levar em consideração a facilidade de ajuste com relação ao sistema em que está sendo utilizado, bem como propiciar a obtenção de propriedades físicas compatíveis com as observadas experimentalmente, caso a aproximação de HartreeFock-Roothaan seja suficiente para representar adequadamente a distribuição eletrônica.

- Número de funções de base a ser utilizado deve corresponder ao de uma série completa, ou tão próximo desta quanto possível. Em outras palavras, 
o cálculo Hartree-Fock não deveria apresentar dependência significativa com relação ao número de funções de base.

- A escolha pelo tipo e número de funções de base também é norteada por um aspecto puramente computacional: a determinação de todas as integrais necessárias ao cálculo de qualquer propriedade do sistema deve ser obtida sem grande dificuldade.

Nas próximas seções serão discutidos aspectos relacionados a estes três critérios.

Tipos de funções de base

\section{Funções Hidrogenóides}

Nos primeiros anos de desenvolvimento computacional dos programas a serem utilizados para resolver as equações de Hartree-Fock, a escolha pelo tipo de função de base recaiu naturalmente sobre as funções hidrogenóides, uma vez que haviam sido obtidas como solução exata da equação de Schrödinger para átomos monoeletrônicos e correspondiam a funções orbitais para esses átomos.

Para átomos mono-eletrônicos com carga nuclear $\mathrm{Z}$ as funções hidrogenóides em coordenadas polares apresentam a seguinte forma:

$$
\chi_{n l m}(r, \theta, \phi)=R_{n l}(r) Y_{l m}(\theta, \phi) \quad \text { equação (1) }
$$

sendo:

$$
R_{n l}(r)=r^{l} \exp \left(-\frac{Z r}{n}\right) \beta_{n+l}^{2 l+l}\left(\frac{2 Z r}{n}\right) \quad \text { equação (2) }
$$

\section{Funções de Slater}

Um dos primeiros casos de funções de base bem sucedidas, no sentido de simplificar a determinação das integrais de energia foram as funções de Slater. Estas funções apresentam uma certa semelhança com as funções hidrogenóides. Sua representação matemática corresponde à apresentada pela Equação 1 porém substituindo-se a parte radial por:

$$
R_{n}(r, \zeta)=N(n, \zeta) r^{n-1} \exp (-\zeta r)
$$

Sendo:

$$
N(n, \zeta)
$$

um fator de normalização correspondendo a

$$
N(n, \zeta)=(2 / \zeta)^{(n+1 / 2)} /[(2 n) !]^{1 / 2}
$$

$\zeta$ é um parâmetro que deverá ser ajustado e função de algum critério predeterminado. Normalmente utilizase o critério de mínima energia para determinar o seu valor. Uma das diferenças entre os dois tipos de funções está relacionada ao tipo de potencial que possibilitou o seu desenvolvimento. As funções hidrogenóides correspondem a soluções da equação de Schrödinger quando o potencial apresenta a forma $V=-Z / r$, enquanto que as funções de Slater são também soluções da equação de Schrödinger, porém para um potencial do tipo $V=-$ $\zeta n / r+[n(n-1)-1(1+1)] /\left(2 r^{2}\right)$. Outro aspecto que diferencia as funções hidrogenóides das funções de Slater está no fato de que as primeiras são funções ortogonais, enquanto que as funções de Slater correspondem a funções nãoortogonais.

\section{Funções Gaussianas}

Funções de Slater não permitem uma resolução analítica e rápida das integrais de dois elétrons de mais de um centro ou ainda integrais de atração nuclear de três centros. Para contornar-se o problema da solução analítica de integrais de energia, funções do tipo gaussiana (Gaussian Type Orbitals - GTO) foram introduzidas. As funções gaussianas podem ser representadas em coordenadas polares pela Equação 1 substituindo-se a função radial por:

$$
R_{n}(r, \alpha)=N(n, \alpha) r^{n-1} \exp \left(-\alpha r^{2}\right) \quad \text { equação (4) }
$$

sendo

$$
N(n, \alpha)=2^{(n+1)} \alpha^{(2 n+1) / 4}[(2 n-) ! !]^{1 / 2}(2 \pi)^{-1 / 4}
$$

Assim como o parâmetro $\zeta$ nas funções de Slater, os parâmetros a são determinados em função de algum critério a ser definido. Como pode ser observado a diferença básica entre a função radial de Slater (Equação 3) e a função radial gaussiana, além do fator de normalização, está no 
termo exponencial que na função gaussiana encontra-se elevado ao quadrado. Esta pequena diferença introduz uma importante propriedade em favor das funções gaussianas, que pode ser enunciado como: o produto de duas ou mais funções gaussianas será sempre uma função gaussiana [4]. Por exemplo, o produto de duas funções gaussianas $G\left(\alpha_{i}, r_{A}\right)$ e $G\left(\alpha_{j}, r_{B}\right)$ centradas nas coordenadas $A=\left(A_{x}, A_{y}, A_{z}\right)$ e $B=\left(B_{x}, B_{y}, B_{z}\right)$, respectivamente, será uma função gaussiana $\mathrm{G}\left(\alpha_{\mathrm{k}}, \mathrm{rC}\right)$ centrada em $\mathrm{C}=(\mathrm{Cx}, \mathrm{Cy}$, $\mathrm{Cz})$. Matematicamente:

$$
G\left(\alpha_{i}, r_{A}\right) \cdot G\left(\alpha_{j}, r_{B}\right)=K \cdot G\left(\alpha_{k}, r_{C}\right) \quad \text { equação }(5)
$$

sendo

$$
K=\exp \left(\frac{a_{i} a_{j}}{a_{i}+a_{j}} \overline{A B}^{2}\right) \quad \text { equação (6) }
$$

e

$$
\alpha_{k}=\alpha_{i}+\alpha_{j}
$$

e o centro C será definido em termos das coordenadas:

$$
\begin{aligned}
C_{x} & =\frac{\alpha_{i} A_{x}+\alpha_{j} B_{x}}{\alpha_{i}+\alpha_{j}} \\
C_{y} & =\frac{\alpha_{i} A_{y}+\alpha_{j} B_{y}}{\alpha_{i}+\alpha_{j}} \\
C_{z} & =\frac{\alpha_{i} A_{z}+\alpha_{j} B_{z}}{\alpha_{i}+\alpha_{j}}
\end{aligned} \quad \text { equação (7) }
$$

Desta forma, a principal razão para a utilidade de funções gaussianas em cálculos de estrutura eletrônica está na facilidade com que produtos de integrais multidimensionais podem ser fatorados e reescritos em termos de centros alternativos. Por exemplo, o cálculo da integral de recobrimento entre as funções $G\left(\alpha_{i}, r_{A}\right)$ e $G\left(\alpha_{j}, r_{B}\right)$ pode ser representado matematicamente por:

$$
\iiint G\left(\alpha_{i}, r_{A}\right) \cdot G\left(\alpha_{j}, r_{B}\right) d_{x} d_{y} d_{z}=K \iiint G\left(\alpha_{k}, r_{C}\right) d_{x} d_{y} d_{z} \text { equação (8) }
$$

sendo que grande vantagem deste tratamento é que a integral a direita pode ser fatorada em termos de:

$$
\iiint G\left(\alpha_{k}, r_{C}\right) d_{x} d_{y} d_{z}=\int_{-\infty}^{\infty} G\left(\alpha_{k}, x_{C}\right) d_{x} \int_{-\infty}^{\infty} G\left(\alpha_{k}, y_{C}\right) d_{y} \int_{-\infty}^{\infty} G\left(\alpha_{k}, z_{C}\right) d_{z} \text { equação (9) }
$$

e cada uma das integrais nas respectivas coordenadas apresentam uma solução conhecida ou seja, convertemos uma integral tripla no produto de três integrais unidimensionais. Está propriedade possibilita o cálculo analítico de integrais de energia necessárias para o cálculo Hartree-Fock.

Funções gaussianas, assim como as funções de Slater, também podem ser representadas em termos de coordenadas cartesianas e consequentemente são chamadas de gaussianas cartesianas e expressas por:

$$
\chi_{l m n}(x, y, z ; \alpha)=N(l, m, n ; \alpha) x^{l} y^{m} z^{n} \exp \left(-\alpha r^{2}\right) \text { equação }
$$

Nesta expressão N $(1, \mathrm{~m}, \mathrm{n} ; \alpha)$ é a constante de normalização para as funções gaussianas em coordenadas cartesianas, $\mathrm{x}$, y e z são as coordenadas eletrônicas cartesianas e 1 , m e $\mathrm{n}$ são conjuntos de números inteiros maiores ou iguais a zero. Estes números não podem ser associados a números quânticos, embora caracterizem a simetria das funções de base, por exemplo:

\begin{tabular}{|l|l|l|l|l|}
\hline 1 & $\mathbf{m}$ & $\mathbf{n}$ & $\mathbf{l + n}+\mathbf{m}$ & simetria \\
\hline 0 & 0 & 0 & 0 & $\mathrm{~s}$ \\
\hline 1 & 0 & 0 & 1 & $\mathrm{p}_{\mathrm{x}}$ \\
\hline 0 & 1 & 0 & 1 & $\mathrm{p}_{\mathrm{v}}$ \\
\hline 0 & 0 & 1 & 1 & $\mathrm{p}_{\mathrm{z}}$ \\
\hline 2 & 0 & 0 & 2 & $\mathrm{~d}_{\mathrm{x}}{ }^{2}$ \\
\hline 0 & 2 & 0 & 2 & $\mathrm{~d}_{\mathrm{v}}{ }^{2}$ \\
\hline 0 & 0 & 0 & 2 & $\mathrm{~d}_{\mathrm{z}}{ }^{2}$ \\
\hline 1 & 1 & 0 & 2 & $\mathrm{~d}_{\mathrm{xy}}$ \\
\hline 1 & 0 & 1 & 2 & $\mathrm{~d}_{\mathrm{xz}}$ \\
\hline 0 & 1 & 1 & 2 & $\mathrm{~d}_{\mathrm{yz}}$ \\
\hline
\end{tabular}

Pode-se verificar que a soma $n+1+m$ produz os números quânticos de momento angular que caracterizam as funções do tipo s, p, d, ...

Um outro aspecto pode também ser verificado em relação as funções gaussianas em coordenadas polares. A combinação dos índices $1, \mathrm{~m}$ e $\mathrm{n}$ produz 6 funções $\mathrm{d}$ diferentes $\left(\mathrm{d}_{\mathrm{xx}}, \mathrm{d}_{y y}, \mathrm{~d}_{z z}, \mathrm{~d}_{x y}, \mathrm{~d}_{\mathrm{xz}}\right.$ e $\left.\mathrm{d}_{\mathrm{yz}}\right)$, enquanto que utilizando-se funções gaussianas em coordenadas polares verifica-se apenas 5 funções diferentes. $\mathrm{Na}$ verdade combinando-se linearmente as funções $\mathrm{d}$ em coordenadas cartesianas verifica-se que uma dessas combinações corresponde a uma função com características de funções s (ex: $3 d_{x x}+3 d_{y y}+3 d_{z z}=N\left(x^{2}+y^{2}+z^{2}\right) \exp \left(-\alpha r^{2}\right)=N r^{2} \exp (-$ $\left.\left.\alpha r^{2}\right)\right)$. O mesmo problema ocorre para funções do tipo $f, g$, etc. é possível, entretanto, utilizar-se funções gaussianas cartesianas do tipo d, f, g, etc. e introduzir-se uma correção que elimine a combinação de funções não desejada. 
Critérios para a adaptação de funções de base

Uma vez definido o tipo de função de base a ser utilizado a pergunta que surge naturalmente é como estas funções poderão ser adaptadas aos sistemas de interesse. Para adaptar-se uma única função ou um conjunto de funções é necessário definir-se critérios de ajuste.

\section{Método Variacional}

Como mencionado anteriormente, um dos critérios mais utilizados para adaptar-se funções de base a um sistema corresponde ao método variacional [5]. De maneira simplificada, se for criada uma função de onda aproximada ( $\Psi)$ para representar o estado eletrônico de um átomo ou molécula, então será obtida uma energia eletrônica dada pela expressão:

$$
\langle E\rangle=\frac{\int \Psi^{*} \hat{H} \Psi d \tau}{\int \Psi^{*} \Psi d \tau}=\frac{\left\langle\Psi^{*}|H| \Psi\right\rangle}{\left\langle\Psi^{*} \mid \Psi\right\rangle} \quad \text { equação (11) }
$$

De acordo com o teorema variacional, a energia $\mathrm{E}$ será sempre maior, ou na melhor das hipóteses igual, a energia exata $\mathrm{E}_{0}$.

$\mathrm{Na}$ prática não conhecemos a energia exata $\mathrm{E}_{0}$. Porém, se forem criadas diferentes funções de onda aproximadas para representar esse mesmo sistema, $\Psi_{1}, \Psi_{2}, \Psi_{3}, \ldots$, o que produzirá energias $\mathrm{E}_{1}, \mathrm{E}_{2}, \mathrm{E}_{3}, \ldots$, respectivamente, então de acordo com o teorema variacional a melhor função de onda será aquela que produzir a menor energia e que certamente estará mais próxima do valor exato da energia E0. Supondo-se que E2 < E1< E3 <..., então $\Psi_{2}$ estará representando mais adequadamente o sistema do que qualquer uma das outras funções de onda e certamente $\mathrm{E}_{2} \geq \mathrm{E}_{0}$.

O método variacional corresponde a um dos critérios mais utilizados para o desenvolvimento e adaptação de funções de base. Ele sugere que pode-se criar qualquer função de onda com qualquer conjunto de base, que o melhor conjunto de base e a melhor função de onda será aquela que proporcionar a menor energia eletrônica para o sistema. Em função desta propriedade pode-se imaginar erroneamente que seria extremamente simples definir-se o melhor tipo de função de base, bem como o número de funções a ser utilizado para construir uma função de onda próxima do valor exato. Entretanto, verifica-se na prática que isto não é verdade e que existe um número infinito de possibilidades de construir-se funções de onda, sendo que vários deles podem levar a energias semelhantes tendendo a um limite inferior. Embora, todas essas possibilidades possam ser consideradas como aproximações adequadas do sistema, deve-se levar em consideração aspectos relacionados ao custo computacional envolvido no cálculo das propriedades do sistema. São razões deste tipo que sugerem que o desenvolvimento de uma função de base deva ser efetuado cuidadosamente e deve-se conhecer quais são as técnicas necessárias para minimizar custos computacionais e como pode-se explorar ao máximo o método variacional.

\section{Teorema de Hellmann-Feynman}

O teorema de Hellmann-Feynman pode ser utilizado como uma alternativa para avaliar-se a qualidade de conjuntos de base e pode ser enunciado da seguinte maneira. Para determinadas funções de onda pode-se verificar que:

$$
\frac{\partial E}{\partial \lambda}=\int \Psi^{*} \frac{\partial \hat{H}}{\partial \lambda} \Psi d \tau \quad \text { equação (12) }
$$

sendo $\lambda$ um parâmetro qualquer do sistema. Qualquer função de onda que satisfaça a Equação 12 é considerada como satisfazendo o teorema de Hellmann-Feynman. Para funções de onda que não satisfazem esse teorema, a Equação 12 é escrita como:

$$
\frac{\partial E}{\partial \lambda}=\int \Psi^{*} \frac{\partial \hat{H}}{\partial \lambda} \Psi d \tau+2 \int \Psi^{*} \hat{H} \frac{\partial \Psi}{\partial \lambda} d \tau \text { equação }
$$

onde o segundo termo a direita da Equação 13 corresponde a um erro na função em relação a esse teorema.

A função de onda exata satisfaz a Equação 12, embora funções de onda distantes do resultado exato também possam obedecê-lo, caso satisfaçam determinadas condições. São estas condições que normalmente se utiliza para ajustar de maneira mais adequada as funções de base.

Um dos parâmetros mais utilizados, corresponde as coordenadas nucleares. Neste caso, as derivadas da energia total do sistema em relação as coordenadas nucleares. Estas derivadas são freqüentemente denominadas de gradientes de energia e são quantidades extremamente importantes na obtenção de geometrias de equilíbrio de sistemas moleculares. Uma vez que são raras as utilizações de funções de base que satisfaçam o teorema de Hellmann- 
Feynman, percebe-se que para a otimização de geometria de uma molécula qualquer, o cálculo do gradiente de energia deve ser realizado pela Equação 13.

Entretanto, a questão de interesse neste caso corresponde a saber quais são as condições necessárias e suficientes para que uma função de onda satisfaça esse teorema. Uma das maneiras de satisfazer a Equação 12 é ajustar variacionalmente o centro das funções de base.

As expressões para as funções de base apresentadas anteriormente (Eqs.2, 3, 4 e 10) não mostraram explicitamente a dependência das funções de base com os respectivos centros, uma vez que foram escritas de forma simplificada e consideraram que as funções de base estariam localizadas no centro de um sistema de coordenadas. Porém, quando trabalha-se com moléculas, coloca-se funções de base sobre os núcleos de cada átomo. Desta forma, considerando-se como exemplo funções gaussianas cartesianas, uma representação mais precisa da Equação 10 seria:

$$
\chi_{l m n}(x, y, z ; \alpha)=N(l, m, n ; \alpha)(x-X)^{\prime}(y-Y)^{m}(z-Z)^{n} \exp \left(-\alpha|\vec{r}-\vec{R}|^{2}\right) \quad \text { equação }
$$

onde as coordenadas cartesianas maiúsculas correspondem às coordenadas do centro em que a respectiva função gaussiana foi colocada. Usualmente estas coordenadas correspondem às coordenadas nucleares. Formalmente, entretanto, não existe nada que diga que as funções de base devam ficar sobre os núcleos atômicos e desta forma, o centro de cada uma das funções de base podem ser considerados como parâmetros variacionais.

A flexibilização da localização do centro das funções de base corresponde a uma condição necessária e suficiente para que o teorema de Hellmann-Feynman seja satisfeito. $\mathrm{O}$ inconveniente no ajuste variacional do centro das funções de base é semelhante ao observado no ajuste dos expoentes dessas funções. A definição da localização do centro de uma função de base será dependente do tipo de molécula, bem como do número e tipo de função de base que estão sendo empregados, o que corresponde a vincular a função de base a um sistema específico. Em outras palavras, perde-se o sentido de transferibilidade do conjunto de base de um sistema molecular para outro. Outro inconveniente está no fato de que os ajustes variacionais de parâmetros não lineares consomem um tempo computacional elevado e o melhor desempenho das funções de base não localizadas sobre os núcleos pode ser compensada por funções de base centradas sobre os núcleos, mas consideravelmente maiores.

Os aspectos técnicos negativos mencionados acima, entretanto, não são suficientes para ofuscar as vantagens de funções de onda que satisfaçam o teorema de HellmannFeynman. Uma destas vantagens está no fato de que otimizações de geometria são parte da rotina dos cálculos de propriedades moleculares e consequentemente dependem do cálculo do gradiente de energia. Para funções de onda satisfazendo o teorema de Hellmann-Feynman o cálculo do gradiente de energia corresponde a determinação da integral representada pela Equação 12. Para funções de onda convencionais que não satisfazem esse teorema o mesmo cálculo deve ser desenvolvido pela Equação 13. Embora não esteja explicitado, computacionalmente o cálculo da primeira integral a direita das Equações 12 ou 13 é extremamente simples de ser realizado, enquanto que o segundo termo da Equação 13 introduz integrais de repulsão eletrônica de mais de dois centros.

Uma vez satisfeito o teorema de Hellmann-Feynman pode-se lançar mão de uma ferramenta alternativa na interpretação dos resultados de propriedades físicas moleculares [9]. Enquanto uma parte significativa das interpretações de tendências químicas está fundamentada na utilização do conceito de energia, a Equação 12 introduz um conceito intuitivamente mais simples de ser utilizado, $o$ conceito de força. Substituindo-se o parâmetro $\lambda$ na Equação 12 pelas coordenadas de um núcleo $\mathrm{A}$ tem-se que:

$$
\nabla_{A} E=\frac{\partial E}{\partial X_{A}} \vec{i}+\frac{\partial E}{\partial Y_{A}} \vec{j}+\frac{\partial E}{\partial Z_{A}} \vec{k}=-\vec{F}_{A}
$$

o gradiente de energia corresponde a força exercida sobre o núcleo atômico A. Desta forma, diversos aspectos relacionados à natureza da ligação química, geometria molecular, reatividade química, mecanismos de movimentos internos, etc. têm sido explorados à luz do conceito de força através da utilização do teorema de Hellmann-Feynman [9]. Uma parte significativa destes trabalhos lançou mão do recurso de otimizar o centro das funções de base em ambientes moleculares. Entretanto, métodos teóricos foram estudados para que conjuntos de base transferíveis fossem desenvolvidos, permitindo que o teorema de Hellmann-Feynman fosse satisfeito. Para comentar-se sobre esses recursos é necessário introduzirse o uso de recursos específicos para o aprimoramento de funções de base em ambiente molecular, o que será explorado posteriormente neste texto.

\section{Outros Métodos}

O método variacional e o teorema de Hellmann-Feynman 
são dois dos mais populares métodos utilizados no desenvolvimento de funções de base e consequentemente de funções de onda confiáveis.

Entretanto, existem outros métodos que podem ser empregados conduzindo ao mesmo objetivo. Alguns desses métodos são [10]:

- teoremas hiperviriais,

- energia local,

- condições de "cu sp",

- matriz densidade exata de primeira ordem,

- recobrimento máximo com funções de onda exatas,

- etc.

Alguns desses métodos não têm sido utilizados pelo fato de necessitarem da solução de integrais tão complexas quanto as integrais de vários centros presentes nos cálculos Hartree-Fock. Outros dependem de definições arbitrárias de regiões do espaço ou de densidades de carga para poderem ser utilizados. Desta forma, optouse por concentrar-se esforços nos dois métodos mais frequentemente empregados na literatura: o método variacional e o teorema de Hellmann-Feynman.

\section{O ajuste variacional de expoentes}

Como mencionado anteriomente, uma função de onda aproximada pode ser construída através da combinação linear de funções de base:

$$
\phi_{i}=\sum_{k=1}^{m} C_{i k} \chi_{k}
$$

Todos os coeficientes desta combinação linear são parâmetros que podem ser modificados de tal maneira que a energia do sistema seja mínima. Por analogia com métodos de regressão, quanto maior o número de parâmetros a ser ajustado para representar uma função qualquer, melhor será a representação dessa função. Portanto, pode-se imaginar que quanto maior o número de funções de base, melhor a representação da função de onda e melhor a energia do sistema. Entretanto, quando utiliza-se um conjunto qualquer de funções de base, podese perguntar se apenas os coeficientes de combinação linear são ajustáveis variacionalmente. A resposta é não, e observando-se melhor a forma matemática das funções de base acima (funções de Slater - Equação 3 e funções gaussianas - Equações 4 e 10) pode-se verificar que existem outros parâmetros que podem ser ajustados de tal maneira que a energia seja minimizada. Funções de Slater e gaussianas dependem, por exemplo, dos parâmetros $\zeta$ e $\alpha$, respectivamente. Quando coloca-se um conjunto de funções de Slater sobre um átomo, qual deve ser o valor dos expoentes $\zeta$ de cada função? E se forem utilizadas funções gaussianas, qual deve ser o valor dos expoentes $\alpha$ ? A resposta é simples, pode-se otimizar esses parâmetros de tal maneira que a energia do sistema onde foram otimizados seja mínima, ou seja, tanto $\zeta$, quanto $\alpha$ são parâmetros que podem ser tratados variacionalmente.

$\mathrm{Na}$ Tabela 1 pode-se encontrar diferentes conjuntos de funções de base do tipo Slater e gaussianas para representar a distribuição eletrônica do átomo de Be bem com as respectivas energias eletrônicas. Os resultados apresentados mostram alguns aspectos interessantes. $\mathrm{O}$ primeiro com relação ao número de funções de base. Por exemplo, verifica-se, como esperado, que quanto maior o número de funções de base de Slater ou gaussianas, melhor a energia do sistema. Um outro aspecto bastante significativo está relacionado com o resultado final da energia eletrônica quando compara-se cálculos realizados com funções de Slater com cálculos feitos com funções gaussianas. O número de funções de Slater para atingirse uma determinada energia é significativamente inferior quando comparado com funções gaussianas. Portanto, para atingir-se um nível de precisão obtido com um certo conjunto de base de Slater, deve-se utilizar um maior conjunto de funções gaussianas.

Tabela 1: Funções de Slater e Gaussianas para o átomo de Be.

\begin{tabular}{|l|l|l|l|l|l|}
\hline Função & GTO $^{(a)}$ & Função & STO $^{(\mathbf{b})}$ & Função & STO $^{(\mathbf{b})}$ \\
\hline $\mathrm{s}$ & 24528,7211 & $1 \mathrm{~s}$ & 5.7531 & $\mathrm{~s}$ & 11.6273 \\
\hline $\mathrm{s}$ & 10121.3264 & $1 \mathrm{~s}$ & 3.7156 & $\mathrm{~s}$ & 7.8469 \\
\hline $\mathrm{s}$ & 4176.3795 & $3 \mathrm{~s}$ & 9.9670 & $\mathrm{~s}$ & 5.2956 \\
\hline $\mathrm{s}$ & 1723.3063 & $3 \mathrm{~s}$ & 3.7128 & $\mathrm{~s}$ & 3.5738 \\
\hline $\mathrm{s}$ & 711.0907 & $2 \mathrm{~s}$ & 4.4661 & $\mathrm{~s}$ & 2.4118 \\
\hline $\mathrm{s}$ & 293.4185 & $2 \mathrm{~s}$ & 1.2919 & $\mathrm{~s}$ & 1.6277 \\
\hline $\mathrm{s}$ & 121.0738 & $2 \mathrm{~s}$ & 0.8555 & $\mathrm{~s}$ & 1.0984 \\
\hline $\mathrm{s}$ & 49.9589 & & & $\mathrm{~s}$ & 0.7413 \\
\hline $\mathrm{s}$ & 20.6146 & & & $\mathrm{~s}$ & 0.5003 \\
\hline $\mathrm{s}$ & 8.5062 & & & $\mathrm{~s}$ & 0.337 \\
\hline $\mathrm{s}$ & 3.5099 & & & & \\
\hline $\mathrm{s}$ & 1.4483 & & & & \\
\hline $\mathrm{s}$ & 0.5976 & & & & \\
\hline $\mathrm{s}$ & 0.2466 & & & & \\
\hline $\mathrm{s}$ & 0.1018 & & & & \\
\hline $\mathrm{s}$ & 0.0420 & & & & \\
\hline $\begin{array}{l}\text { Energia } \\
\text { (u.a.) }\end{array}$ & -14.57302 & & & & \\
\hline
\end{tabular}

(a) Custodio, R. et al., Can. J. Chem. 1992, 70: 580. (b) Bunge, C.F., Barrientos, J.A. e Bunge, A.V., "Atomic Data and Nuclear Data Tables" 1993, 53: 113 
A Tabela 1 mostra ainda que os parâmetros variacionais diferem significativamente quando o número de funções é alterado. Pode-se verificar que existe uma tendência do conjunto de base de se deslocar para a região mais próxima do núcleo ( as funções com maior expoentes são as funções que localizam a distribuição eletrônica mais próxima da região nuclear e vice-versa). Este é um aspecto extremamente significativo a ser considerado quando se modela um conjunto de funções de base. A necessidade de conjuntos de base muito grandes está relacionada a descrição da distribuição eletrônica na região próxima dos núcleos e as grandes variações observadas na energia eletrônica para pequenas variações nos expoentes que descrevem essa região. Algumas técnicas sugerem que os elétrons presentes na região interna possam ser substituídos por determinadas funções potenciais que simulem a presença desses elétrons, mas que dispensam funções de base para representar a nuvem eletrônica nessa região.

Otimização de expoentes em diferentes ambientes

Da mesma maneira que os expoentes apresentados na Tabela 1 foram ajustados variacionalmente no ambiente atômico, pode-se efetuar a mesma otimização dos expoentes dos conjuntos de base em sistemas moleculares e adequá-los a esse ambiente. A tendência geral em cálculos moleculares é que cada átomo na molécula apresente seu conjunto de base e que, de acordo com o método Hartree-Fock-Roothaan, os mesmos sejam misturados para produzir os orbitais moleculares. Os expoentes das funções de base podem ser otimizados para ajustar de maneira mais adequada a distribuição eletrônica no ambiente molecular. Desta forma, um mesmo número de funções de base que esteja descrevendo um átomo, certamente apresentará diferentes expoentes dependendo se os mesmos foram ajustados no átomo isolado ou em ambiente molecular. Esta diferença entre os expoentes otimizados em ambiente atômico e molecular caracteriza o que se denomina na literatura de expoentes otimizados em ambiente atômico e expoentes otimizados em ambiente molecular.

A Tabela 2 mostra um exemplo para o átomo de $\mathrm{F}$ de expoentes de um conjunto de funções de Slater otimizados no átomo isolado e otimizados na molécula de $\mathrm{F}_{2}$. Este exemplo mostra, como esperado, que os expoentes ajustados no ambiente atômico diferem daqueles ajustados no ambiente molecular. Deve-se lembrar que os expoentes estão sendo otimizados em relação ao critério de mínima energia e que as interações sofridas pelas partículas no ambiente molecular são diferentes do ambiente atômico.

Tabela 2: Expoentes do átomo de $\mathrm{F}$ otimizados no átomo neutro (estado: ${ }^{2}$ P) e na molécula $F_{2}$ (estado: ${ }^{1} \sum_{\mathrm{g}}^{+}$, Req=2,680 u.a.).

\begin{tabular}{|l|l|l|}
\hline Expoentes & $\begin{array}{l}\text { Otimizados no } \\
\text { átomo (FBOA) }\end{array}$ & $\begin{array}{l}\text { Otimizados na } \\
\text { molécula (FBOM) }\end{array}$ \\
\hline$\varsigma_{1 \mathrm{~s}}$ & 8,6501 & 8,6504 \\
\hline$\varsigma_{2 \mathrm{~s}}$ & 2,5639 & 2,5776 \\
\hline$\varsigma_{2 \mathrm{p} \sigma}$ & 2,5498 & 2,4934 \\
\hline$\varsigma_{2 \mathrm{p} \pi}$ & 2,5498 & 2,5688 \\
\hline E/u.a. & $-197,87409$ & $-197,87694$ \\
\hline
\end{tabular}

Baseado neste exemplo pode-se imaginar uma situação mais complexa. O esforço computacional no processo de otimização dos expoentes de um mesmo conjunto de base em ambiente atômico e no ambiente de uma molécula diatômicahomonuclearnão deve diferirsignificativamente. A etapa que deverá gastar maior tempo computacional deverá ser o tempo gasto com a montagem da matriz de Fock, o processo de convergência do cálculo HartreeFock e o maior número de integrais a ser calculado no ambiente molecular. Porém, supondo-se uma molécula mais complexa, pode-se verificar que o tempo despendido no processo de otimização dos expoentes nesse ambiente poderá tornar-se computacionalmente proibitivo. Se cada átomo na molécula precisa ter seus expoentes otimizados, o custo computacional deverá crescer exponencialmente. Para se ter uma idéia, pode-se imaginar a otimização de cinco funções de Slater para um átomo de $\mathrm{C}$ no estado fundamental correspondendo a funções $1 \mathrm{~s}, 2 \mathrm{~s}, 2 \mathrm{p}_{\mathrm{x}}, 2 \mathrm{p}_{\mathrm{y}} \mathrm{e}$ $2 \mathrm{p}_{\mathrm{z}}$ e a otimização dos expoentes dos átomos de carbono em uma molécula como o propano que contém três átomos de carbono. $\mathrm{O}$ número de funções de base a ser otimizado na molécula, desconsiderando-se propriedades de simetria e as 8 funções 1s dos átomos de hidrogênio, seria de 15 funções de base. O número das integrais de energia a ser calculado esta diretamente relacionado ao número de funções de base a ser utilizado. Enquanto as integrais de energia cinética e atração núcleo-elétron crescem na ordem de $n^{2}$ (mais precisamente: $n(n+1) / 2$ ), onde $\mathrm{n}$ representa o número de funções de base, o número de integrais de repulsão elétron-elétron cresce na ordem de $n^{4}$ (ou seja, $\left(n^{4}+2 n^{3}+3 n^{2}+2 n\right) / 8$ ). Pode-se, portanto, verificar que o número de integrais de repulsão elétronelétron a ser calculado no propano, será de 7260 , enquanto que no ambiente atômico é de apenas 120. Entretanto, este não é o agravante mais sério e qualquer cálculo molecular deverá manipular esse número significativo de integrais. Uma parte importante do problema está na maneira como são variados os expoentes para atingirse o mínimo de energia. Os métodos mais eficientes e 
utilizados na minimização da energia estão baseados em informações contidas no gradiente de energia em relação aos parâmetros a serem otimizados. A obtenção dos gradientes de energia em relação aos expoentes das funções de base através de métodos analíticos produzem expressões matemáticas muito mais complexas do que aquela obtida para a energia eletrônica do sistema. Em casos simplificados, pode-se determinar os gradientes de energia através de métodos numéricos. Neste caso, para cada expoente a ser otimizado deve-se realizar uma série de 3 a 5 cálculos Hartree-Fock para possibilitar o cálculo de uma derivada de energia em relação a esse expoente, ou seja, pode-se traduzir isto como um esforço computacional gigantesco quando comparado com cálculos atômicos e que o processo de otimização dos expoentes em ambiente molecular está restrito atualmente a sistemas moleculares pequenos.

Como resolver o problema dos expoentes das funções de base em ambiente molecular? A sugestão mais simples é procurar verificar qual a perda de energia se forem utilizados os expoentes otimizados para o átomo no cálculo molecular. A Tabela 2 mostra o resultado da transferência do conjunto de base do átomo de $\mathrm{F}$ para a molécula de $\mathrm{F}_{2}$ e o respectivo valor de energia para o mesmo cálculo com expoentes otimizados na molécula. Pode-se observar que a perda de energia é relativamente pequena. Uma alternativa para minimizar essa perda de energia seria otimizar-se os expoentes de um conjunto de base maior em ambiente atômico e transferi-lo para o ambiente molecular. A idéia da transferibilidade, embora não corresponda ao conjunto de base mais adequado, é funcional e tornou-se a tendência de quase todos os cálculos Hartree-Fock. De uma certa forma, o controle dos resultados moleculares foi transferido praticamente para o controle no número de funções de base a ser utilizado. Funções de base de diferentes tamanhos seriam otimizadas em ambiente atômico e transferidas para ambientes moleculares e o conjunto que apresentasse resultados mais adequados em termos de energia e computacionalmente mais viáveis seria utilizado para diferentes cálculos moleculares. Esta observação deve ser avaliada com cautela, uma vez que os cálculos moleculares apresentam exigências que não são observadas em cálculos atômicos, uma vez que a nuvem eletrônica estará sob a influência de mais de um núcleo atômicos e portanto, deformando a nuvem eletrônica e um campo não esférico e consequentemente afetando significativamente energias e outras propriedades eletrônicas. Entretanto, aspectos mais relacionados aos cálculos moleculares serão discutidos após a apresentação de outros critérios para otimizar-se funções de onda.

\section{Séries geométricas}

Uma maneira engenhosa de minimizar o tempo computacional otimizando-se os expoentes dos conjuntos de base foi sugerido por Ruedenberg [6]. Observando a tendência de expoentes otimizados de funções de base gaussianas em ambiente atômico, Ruedenberg verificou que os mesmos poderiam ser representados por uma série geométrica do tipo:

$$
\alpha_{i}(k)=a(k) b(k)^{i-1}
$$$$
\text { equação (17) }
$$

onde a e b são parâmetros a serem ajustados variacionalmente ou por método de mínimos quadrados em relação ao conjunto de base original para as funções de simetria $\mathrm{k}$ e i $=1,2,3, \ldots$ correspondendo ao número de funções de base na série original. Desta forma, os expoentes de um conjunto de funções do tipo s poderiam ser aproximados pela relação acima desde que fossem conhecidos os valores de $\mathrm{a}, \mathrm{b}$ e o número de funções para este conjunto de base.

Funções de base definidas em termos da Eq.17 foram denominadas de séries even-tempered. $\mathrm{O}$ aspecto mais significativo desta aproximação está no fato de que se essa representação for adequada para representar um conjunto de base, independente do seu tamanho, apenas dois parâmetros ( $\mathrm{e}$ e b) precisam ser definidos para caracterizar aproximadamente a série de expoentes. $\mathrm{Na}$ Tabela 3 encontram-se os expoentes de um conjunto de base para o átomo de $\mathrm{He}$ constituídos por 15 funções gaussianas do tipo s completamente otimizados e otimizados através do uso da série geométrica even-tempered (Eq.17). A diferença de energia entre os dois cálculos é desprezível.

Tabela 3: Expoentes de um conjunto de base para o átomo de $\mathrm{He}$ constituidos por 15 funções gaussianas do tipo s completamente otimizados e otimizados através do uso da série geométrica eventempered. Os dois últimos valores negativos correspondem a energia total obtida em nível Hartree-Fock com os dois conjuntos de base.

\begin{tabular}{|l|l|}
\hline $\begin{array}{l}\text { Expoentes } \\
\text { otimizados }^{(a)}\end{array}$ & $\begin{array}{l}\text { Série “even } \\
\text { tempered" }^{(b)}\end{array}$ \\
\hline 15546.27532 & 15648.41867 \\
\hline 6692.22470 & 6736.36094 \\
\hline 2880.81039 & 2899.88143 \\
\hline 1240.10607 & 1248.34645 \\
\hline 533.83002 & 537.39054 \\
\hline 229.79848 & 231.33690 \\
\hline 98.92164 & 99.58634 \\
\hline 42.58292 & 42.87012 \\
\hline 18.33072 & 18.45481 \\
\hline 7.89085 & 7.94446 \\
\hline
\end{tabular}




\begin{tabular}{|l|l|}
\hline 3.39678 & 3.41994 \\
\hline 1.46221 & 1.47222 \\
\hline 0.62944 & 0.63376 \\
\hline 0.27095 & 0.27282 \\
\hline 0.11663 & 0.11744 \\
\hline
\end{tabular}

a) Energia $=-2.8616791147$ u.a.; b) Energia $=-2.8616791128$ u.a..

Posteriormente, com o intuito de aumentar o grau de flexibilidade da série even-tempered, foram desenvolvidas outras séries envolvendo um número maior de parâmetros variacionais, como a série desenvolvida por Huzinaga denominada de well-tempered [7] e representada por:

$$
\alpha_{i}(k)=a(k) b(k)^{i-1}\left[1+y\left(\frac{i}{K}\right)^{\delta(k)}\right]
$$

Nesta série verifica-se um aumento significativo no número de parâmetros variacionais de dois (na série eventempered: a e b) para quatro (na série well-tempered: $a, b$, $\gamma$ e $\delta$ ). Com o intuito de reduzir o esforço computacional no processo de otimização dos parâmetros variacionais, Huzinaga sugeriu que a mesma série de parâmetros deveria ser usada para todos os tipos de funções de base, $\mathrm{s}, \mathrm{p}, \mathrm{d}, \ldots$, ou seja, se o conjunto de base for aumentado por funções de momento angular maior do que os já existentes, deve-se utilizar a mesma série de parâmetros definidos para a série.

Estas observações obtidas em relação as séries geométricas culminaram com a hipótese de que, em princípio, seria possível construir-se um único conjunto de base constituído por funções do tipo $s, p, d, \ldots$ que pudesse ser aplicado a qualquer átomo da tabela periódica. Este conjunto de base foi denominado de base universal [8].

Embora o método variacional seja considerado em quase todos os processos de adequação de funções de onda, existem outros critérios que não fazem uso do mínimo de energia e que podem nortear o desenvolvimento e/ou adaptação de funções de base em ambientes atômicos e/ou moleculares.

\section{Funções de adicionais}

Em ambiente atômico, a realização de cálculos HartreeFock leva em consideração funções de base com a mesma simetria dos orbitais atômicos ocupados. Em outras palavras, se o cálculo Hartree-Fock para o átomo de C, que possui seis elétrons for realizado, sabe-se que no estado fundamental a sua configuração eletrônica é: $1 \mathrm{~s}^{2} 2 \mathrm{~s}^{2} 2 \mathrm{p}^{2}$. Desta forma, as funções de base a serem utilizadas para um cálculo para esse átomo devem apresentar simetria s e p apenas. Funções de base com momento angular superior não são consideradas, uma vez que não existem orbitais ocupados diferentes de s e p e que funções de base de simetria diferente não se misturam às funções $s$ e $p$. Caso seja considerada a possibilidade de calcular-se algum estado atômico excitado que inclua orbitais atômicos do tipo d ou de qualquer outra simetria, então deve-se proceder a inclusão deste tipo de função de base para uma representação adequada da distribuição eletrônica.

Quando são realizados cálculos para moléculas utilizamse funções de base adaptadas em átomos, ou seja, se um hidrocarboneto como o etano está sendo estudado, utilizam-se funções de base adaptadas para o átomo de carbono e hidrogênio. Sabendo-se que no estado fundamental o átomo de carbono possui apenas orbitais ocupados do tipo s e p e que o hidrogênio apresenta apenas um orbital do tipo s, então, no sistema molecular serão incluídas apenas funções de base do tipo s e p.

Entretanto, a distribuição eletrônica no ambiente molecular éextremamentemaiscomplexa do que no ambiente atômico. O conjunto de funções de base desenvolvido no ambiente atômico não leva em consideração distorções da nuvem eletrônica, característica de sistemas multicêntricos. Com o intuito de descrever mais adequadamente propriedades moleculares e preservar a transferência de funções de base desenvolvidas em ambientes atômicos, observou-se que determinadas funções de base deveriam ser acrescentadas na função de base original, quando aplicada em sistemas moleculares. Estas funções foram classificadas em dois grupos: a) funções de polarização e b) funções difusas.

\section{Funções de polarização}

As funções de polarização auxiliam na descrição das distorções da nuvem eletrônica em ambiente molecular, importantes na descrição das ligações químicas, e correspondem a funções adicionais com momento angular diferente daquele apresentado pela base original. Por exemplo, para o átomo de carbono uma função de base convencional incluiria apenas funções de base do tipo s e p. A inclusão de funções de polarização corresponderia a inclusão de funções do tipo d, f, g, etc.

O número de funções de polarização empregado em cálculos Hartree-Fock em geral é reduzido, embora sua 
influência seja significativa na tendência de determinadas propriedades moleculares. Para átomos do $2^{\circ}$ e $3^{\circ}$ períodos da tabela periódica as funções de base mais frequentemente empregadas na literatura utilizam de uma a três funções de polarização do tipo d.

A definição dos expoentes disponíveis na literatura de funções de polarização é normalmente obtida através do método variacional. $\mathrm{O}$ ajuste dos expoentes, em geral, é efetuado em ambiente molecular. Selecionase um conjunto de moléculas simples e representativo contendo o elemento para o qual está sendo ajustada a correspondente função de polarização e utiliza-se uma média dos expoentes obtidos para todas as moléculas. Este valor médio deve ser tal que a diferença de energia entre o expoente otimizado e a energia calculada com o expoente médio seja mínima.

Uma outra maneira sistemática de introduzir-se funções de polarização em cálculos Hartree-Fock utiliza o teorema de Hellmann-Feynman. A energia obtida neste processo pode ser escrita como função das coordenadas nucleares, $R_{A}$, e de uma série de parâmetros não lineares envolvidos na função de onda representados por $\mathrm{x}_{\mathrm{r}}$. Uma vez que o teorema de Hellmann-Feynman está associado ao ajuste do centro das funções de base considerar-se-á apenas estes parâmetros não lineares no conjunto $\mathrm{x}_{\mathrm{r}}$. Desta forma, a função de onda obtida através de um conjunto de base qualquer normalmente não satisfaz o teorema de Hellmann-Feynman e a Equação13 será escrita explicitamente em termos destes parâmetros como:

$$
\vec{F}_{A}=\int \Psi^{*} \frac{\partial \hat{H}}{\partial \lambda} \Psi d \tau-\sum_{r} \Delta_{r} \frac{\partial x_{r}}{\partial R_{A}} \quad \text { equação (19) }
$$

sendo:

$$
\Delta_{r}=4 \sum_{i} C_{i r} \sum_{s} C_{i s}\left(F_{r^{\prime} \mathrm{s}}-\varepsilon_{i} S_{r^{\prime} s}\right) \quad \text { equação }(20)
$$

elementos da expressão para $\Delta \mathrm{r}$ assemelham-se a equação secular. A diferença corresponde a utilização das derivadas da função de base r representadas aqui por r'. Pode-se dizer, então, que se em um cálculo Hartree-Fock empregando um conjunto de base qualquer incluir a derivada dessa função de base, então o termo $\Delta \mathrm{r}$ será igual a zero $[9,11]$. Entretanto, se as derivadas das funções de base não forem incluídas o termo $\Delta$ r será diferente de zero e o teorema de Hellmann-Feynman não será obedecido.

De que maneira esta informação pode auxiliar na inclusão das funções de polarização? A resposta é simples se funções gaussianas estiverem sendo utilizadas. Derivando-se a Equação 14 em relação as coordenadas do centro da função de base verifica-se que o derivada de funções gaussianas do tipo s serão funções do tipo $\mathrm{p}$ com mesmo expoente da função s de partida. A derivada de funções do tipo p serão funções gaussianas do tipo $\mathrm{d}$ com mesmos expoentes das funções do tipo p e assim suscessivamente. Por exemplo:

$$
\chi_{\text {GTO }}\left(\alpha ; r_{A}\right)=N \exp \left(-\alpha r_{A}^{2}\right) \quad \text { equação }(21)
$$

e a derivada desta função em relação a componente $x$ será:

$$
\frac{\partial \chi_{\text {GTO }}\left(\alpha ; r_{A}\right)}{\partial X_{A}}=N^{\prime} X_{A} \exp \left(-\alpha r_{A}^{2}\right) \quad \text { equação }(22)
$$

que corresponde a uma função px. Desta forma, funções de polarização podem ser consideradas como derivadas da função de base de partida. Em princípio, deve-se incluir as derivadas de todas as funções de base presentes. Entretanto, isto encareceria significativamente os cálculos moleculares. Através de diversos testes verificou-se que em geral o erro associado ao teorema de HellmannFeynman está localizado em determinadas regiões de valores de expoentes, regiões estas normalmente próximas $\mathrm{da}$ valência [12]. Consequentemente, em função da necessidade de economia com o número de funções de base, apenas as derivadas das funções de base responsáveis pelos maiores desvios do teorema de Hellmann-Feynman são consideradas.

$\mathrm{Na}$ prática, uma maneira correta de saber-se quais funções de polarização devem ser incluídas é calculando-se o termo $\Delta \mathrm{r}$ para cada função de base e então, elegendo-se os valores mais significativos, incluir-se as derivadas das respectivas funções de base em um novo cálculo HartreeFock. Embora os cálculos de $\Delta \mathrm{r}$ não sejam tão complexos quanto a própria equação secular, pode-se substituir este processo formal por um método mais lento, mas possível de ser realizado com qualquer programa Hartree-Fock que permita o cálculo de gradientes de energia e o de campos elétricos sobre os núcleos atômicos.

Quando se otimiza a geometria de uma molécula, utilizase o gradiente de energia para a busca do mínimo de energia. $\mathrm{Na}$ geometria de equilíbrio o gradiente deve ser muito próximo de zero. Se a função de onda não satisfizer o teorema de Hellmann-Feynman, o gradiente de energia será diferente da força de Hellmann-Feynman. Enquanto a primeira quantidade é igual a zero na geometria de equilíbrio, a segunda não é. Pode-se, desta forma, incluir as derivadas das funções de base existentes e otimizarse a geometria molecular toda vez que uma nova função 
de polarização for incluída até que a força de HellmannFeynman seja igual ao gradiente de energia e que na geometria de equilíbrio será igual ou próximo de zero. Esta igualdade implica no fato de que $\Delta \mathrm{r}=0$ ou $\Delta \mathrm{r} \rightarrow 0$.

O cálculo das forças de Hellmann-Feynman é trivial. Conceitos básicos de eletrostática mostram que a força eletrostática $\overrightarrow{\mathrm{F}}_{\mathrm{A}}$ que atua sobre um núcleo $\mathrm{A}$ será igual a carga nuclear deste núcleo, $Z_{\mathrm{A}}$, multiplicada pelo campo elétrico atuando sobre o mesmo, $\overrightarrow{\mathrm{E}}_{\mathrm{A}}$, ou seja:

$$
\overrightarrow{\mathrm{F}}_{\mathrm{A}}=\mathrm{Z}_{\mathrm{A}} \overrightarrow{\mathrm{E}}_{\mathrm{A}} \quad \text { equação (23) }
$$

O campo elétrico é um operador monoeletrônico, extremamente simples de ser calculado e presente na maior parte dos pacotes em uso para cálculos mecânicoquânticos. Assim, uma vez que as forças de HellmannFeynman tendem a zero na geometria de equilíbrio, deve-se verificar se o valor do campo elétrico tenderá a zero nas mesmas condições. Uma vez que o processo de otimização de geometria implica em um gradiente próximo a zero, o valor do gradiente deverá corresponder a um valor menor ou igual ao limite estabelecido no processo de otimização de geometria $[12,13]$. Todo este processo de ajuste da função de base pode ser realizado em moléculas diatômicas ou triatômicas e posteriormente transferir-se o conjunto de base ajustado para moléculas mais complexas.

A utilização das funções de polarização pode modificar significativamente $\mathrm{o}$ valor absoluto ou a tendência das propriedades calculadas. A escolha a ser efetuada depende da qualidade dos resultados a serem obtidos, bem como da disponibilidade computacional. A inclusão das funções de polarização tende a aumentar o conjunto de base significativamente, principalmente em moléculas com elevado número de átomos. Embora tenham sido comentados dois processos diferentes de obtenção de funções de polarização, pode-se ainda incorporar outras propriedades de fácil obtenção em cálculos Hartree-Fock, permitindo um melhor ajuste da função de onda.

\section{Funções difusas}

Um outro conjunto de funções usualmente incluído em cálculos moleculares é o das funções difusas. Ao contrário das funções de polarização que alteram significativamente diversas propriedades moleculares, este conjunto é normalmente necessário para o cálculo de propriedades de ânions. Como mencionado anteriormente, as funções de base são frequentemente desenvolvidas em sistemas atômicos neutros. Quando são utilizadas em sistemas aniônicos, a nuvem eletrônica desses sistemas apresenta uma tendência à expansão, além do que, a descrição do comportamento deste elétron adicional não havia sido contemplada na obtenção do conjunto de base atômica. Uma vez que a facilidade com que a distribuição eletrônica tem de expandir-se está diretamente relacionada ao conjunto de base utilizado, pode-se verificar que o conjunto de base original deve ser aumentado na região de valência, ou seja, devem ser incluídas funções de base do mesmo tipo das funções já existentes, mas com expoentes que descrevam melhor a região de valência.

Para exemplificar, se para um determinado átomo for utilizado um conjunto de base contendo 9 funções s e 5 funções $p$, as funções difusas a serem incluídas deverão ser do tipo s e p com expoentes menores do que os de qualquer função já presente na base $(9 s 5 p)$.

A escolha pelos expoentes das funções difusas pode ser feito variacionalmente em ânions monoatômicos ou, da mesma forma que para funções de polarização, em sistemas representativos de moléculas. No caso do ajuste variacional, apenas os expoentes das funções difusas são otimizados, enquanto que o conjunto de base original permanece inalterado.

Uma maneira simplificada de obter-se expoentes de funções difusas considera que a função de base na região de valência comporta-se como uma série geométrica [14]. Assim, pode-se determinar o expoente das funções difusas através da expressão:

$$
\alpha_{i+1}(k)=\frac{\alpha_{i}^{2}(k)}{\alpha_{i-1}(k)} \quad \text { equação }(24)
$$

Nesta expressão necessita-se dos dois menores expoentes de um determinado tipo de função de base $\mathrm{k}, \alpha_{\mathrm{i}}$ e $\alpha_{\mathrm{i}-1}$, sendo $\alpha_{i}>\alpha_{i-1}$. O expoente $\alpha_{i+1}$ corresponde ao valor do expoente da função difusa do tipo k. Caso seja necessário incluir-se mais funções difusas para a função de simetria $\mathrm{k}$, pode-se utilizar a mesma relação empregando-se os dois menores expoentes desta espécie considerando-se também o expoente da função difusa determinado previamente. Este processo pode ser repetido indefinidamente e gerar tantas funções difusas quantas forem necessárias.

Na Tabela 4 encontra-se um conjunto de base (9s5p) para o átomo de oxigênio. Para efetuar-se o cálculo do ânion Ocom esse conjunto de base é necessário incluir-se funções difusas. Desta forma, as funções difusas adicionais serão 
do tipo s e p. O expoente da função difusa do tipo s será obtido através dos menores expoentes do tipo s (ver Tabela 4) com a equação $24: \alpha_{10}(\mathrm{~s})=0,2846^{2} / 0,9398=8,6186 \mathrm{x}$ $10^{-2}$. Para as funções do tipo $\mathrm{p}$ o expoente da primeira função difusa será: $\alpha_{6}(p)=0.2137^{2} / 0.7171=6,3684 \times 10^{-2}$.

Tabela 4: Expoentes do conjunto de base gaussiana (9s5p) descontraído para o átomo de $\mathrm{O}$.

\begin{tabular}{|l|l|l|l|}
\hline & $\boldsymbol{\alpha}(\mathbf{s})$ & & $\boldsymbol{\alpha}(\mathbf{p})$ \\
\hline$s_{1}$ & 7817,0000 & $p_{1}$ & 35,1800 \\
\hline$s_{2}$ & 1176,0000 & $p_{2}$ & 7,9040 \\
\hline$s_{3}$ & 273,2000 & $p_{3}$ & 2,3050 \\
\hline$s_{4}$ & 81,1700 & $p_{4}$ & 0,7171 \\
\hline$s_{5}$ & 27,1800 & $p_{5}$ & 0,2137 \\
\hline$s_{6}$ & 9,5320 & & \\
\hline$s_{7}$ & 3,4140 & & \\
\hline$s_{8}$ & 0,9398 & & \\
\hline$s_{9}$ & 0,2846 & & \\
\hline
\end{tabular}

O critério para a escolha de quantas e de que tipo de funções difusas devem ser incluídas pode ser obtido através da comparação do efeito destas funções sobre uma ou várias propriedades quaisquer calculadas com e sem funções difusas.

\section{Métodos de contração}

Uma observação dos conjuntos de base disponíveis na literatura, predominantemente conjuntos gaussianos, mostram que um número considerável de primitivas é normalmente utilizado para obtenção de propriedades atômicas ou moleculares. No início dos desenvolvimentos de pacotes computacionais, o problema de espaço em disco para armazenar principalmente integrais de dois elétrons era extremamente reduzido mesmo para moléculas pequenas. Para minimizar o uso de espaço em disco foram desenvolvidas técnicas denominadas de métodos de contração de conjuntos de base. Em poucas palavras, contração de um conjunto de base corresponde a formar grupos com algumas das funções do conjunto de base original e utilizar esses grupos no cálculo de propriedades atômicas e moleculares. Por conveniência para diferenciar as funções de base contraídas das não contraídas, convencionou-se denominar cada função de base não contraída como primitiva.

As duas exigências para a contração de um conjunto de base podem ser resumidas da seguinte maneira:
- o número de grupos de funções deve ser significativamente menor do que o número de primitivas e

- após o processo de contração, os valores de propriedades, tais como energia eletrônica, momento de dipolo, energias orbitais, etc., devem produzir valores muito próximos dos valores obtidos com o conjunto de base não contraído.

O primeiro item justifica-se pelo fato de que espera-se principalmente uma redução nos custos computacionais em relação ao armazenamento de integrais de dois elétrons e em menor extensão a ordem das matrizes envolvidas ( ex: matriz de energia cinética, matriz de Fock, etc.). Portanto, se um sistema como He está sendo representado por um conjunto de 6 funções s, pode-se formar diferentes agrupamentos dessas funções, por exemplo 3 grupos de funções s. Desta forma, enquanto que para o conjunto de funções não contraídas é necessário reservar-se espaço para $6^{4}$ integrais de dois elétrons, para o conjunto contraído, deve-se reservar espaço para $3^{4}$.

O segundo item sugere que quando se efetua a contração de um conjunto de base tem-se necessariamente uma perda de precisão em relação ao conjunto descontraído. Um cálculo Hartree-Fock para o átomo de He com 6 funções gaussianas do tipo s será realizado com o ajuste variacional de 6 coeficientes de combinação linear das 6 primitivas utilizadas. Entretanto, agrupando-se as 6 primitivas em três conjuntos de funções, o número de coeficientes de combinação linear a serem ajustados variacionalmente será de apenas 3 coeficientes. Como mencionado anteriormente, quanto maior o número de parâmetros variacionais, mais próximo se estará da função de onda exata e consequentemente, melhor a descrição da energia do sistema e de outras propriedades.

A grande preocupação dos métodos empregados para contrair funções de base está depositada sobre estes dois aspectos, a maior redução possível do número de grupos de funções com o menor desvio possível das propriedades calculadas em relação aos cálculos com bases descontraídas.

Dos métodos disponíveis na literatura, existem basicamente três possíveis de serem aplicados: a) o método de contração de Dunning [15], b) o método de Raffenetti [16] e c) o método de orbitais naturais [17]. 
O método de contração de Raffenetti

A idéia de contração de um conjunto de base é um reflexo do grau de arbitrariedade na definição do próprio conjunto de base. A escolha de funções gaussianas ou de Slater ou de qualquer outro tipo de função, corresponde a escolhas baseadas na qualidade dos resultados desejados e minimização dos custos computacionais. Quando se realiza um cálculo Hartree-Fock para um átomo qualquer com um conjunto de base arbitrário constituído por um certo número de primitivas, pode-se perguntar se os orbitais atômicos resultantes do cálculo Hartree-Fock não poderiam ser utilizados como funções de base para a realização de um cálculo molecular. Este é o método conhecido como método de contração de Raffenetti [16].

A melhor maneira de demonstrar-se esta idéia é através de um exemplo. Na Tabela 4 tem-se os expoentes de um conjunto de bases gaussianas descontraído constituído por (9s5p) primitivas. Quando utiliza-se este conjunto de base no cálculo Hartree-Fock do átomo de oxigênio em seu estado fundamental obtém-se os orbitais atômicos desse átomo. A Tabela 5 mostra os coeficientes de combinação linear dos orbitais ocupados obtidos como misturas das funções (9s5p). Pode-se realizar um outro cálculo para o mesmo átomo utilizando-se agora, não o conjunto de base (9s5p), mas os orbitais atômicos calculados como conjunto de base. Desta forma, o primeiro orbital s ocupado (primeira coluna da Tabela 5) seria a primeira função de base, o segundo orbital s ocupado (segunda coluna da Tabela 5) seria a segunda função de base, e o primeiro orbital p ocupado (terceira coluna da Tabela 5) seria a terceira função de base. Ao invés de realizar-se o cálculo atômico com o conjunto $(9 \mathrm{~s} 5 \mathrm{p})$ pode-se realizar o mesmo com o conjunto [2s1p].

Tabela 5: Coeficientes de combinação linear obtidos pelo método Hartree-Fock para o átomo de $\mathrm{O}$ no estado fundamental $\left({ }^{3} \mathrm{P}\right) \mathrm{com}$ conjunto de base gaussiana (9s5p) dados na Tabela 4.

\begin{tabular}{|l|l|l|l|l|l|}
\hline & $\boldsymbol{\varepsilon}_{\mathbf{1}}$ & $\boldsymbol{\varepsilon}_{\mathbf{2}}$ & $\boldsymbol{\varepsilon}_{\mathbf{3}}$ & $\boldsymbol{\varepsilon}_{\mathbf{4}}$ & $\boldsymbol{\varepsilon}_{\mathbf{5}}$ \\
\hline $\begin{array}{l}\text { Auto } \\
\text { valores } \\
\text { / u.a. }\end{array}$ & $-20,6663$ & $-1,2422$ & $-0,6294$ & -0.6294 & -0.6294 \\
\hline & $\mathrm{OA}_{1}$ & $\mathrm{OA}_{2}$ & $\mathrm{OA}_{3}$ & $\mathrm{OA}_{4}$ & $\mathrm{OA}_{5}$ \\
\hline $\mathrm{s}_{1}$ & 0,00118 & $-0,00027$ & 0,00000 & 0,00000 & 0,00000 \\
\hline $\mathrm{s}_{2}$ & 0,00898 & $-0,00206$ & 0,00000 & 0,00000 & 0,00000 \\
\hline $\mathrm{s}_{3}$ & 0,04286 & $-0,00979$ & 0,00000 & 0,00000 & 0,00000 \\
\hline $\mathrm{s}_{4}$ & 0,14391 & $-0,03574$ & 0,00000 & 0,00000 & 0,00000 \\
\hline $\mathrm{s}_{5}$ & 0,35559 & $-0,09509$ & 0,00000 & 0,00000 & 0,00000 \\
\hline $\mathrm{s}_{6}$ & 0,46129 & $-0,19587$ & 0,00000 & 0,00000 & 0,00000 \\
\hline $\mathrm{s}_{7}$ & 0,14129 & $-0,03741$ & 0,00000 & 0,00000 & 0,00000 \\
\hline
\end{tabular}

\begin{tabular}{|l|l|l|l|l|l|}
\hline $\mathrm{s}_{8}$ & $-0,00058$ & 0,59568 & 0,00000 & 0,00000 & 0,00000 \\
\hline $\mathrm{s}_{9}$ & 0,00139 & 0,52575 & 0,00000 & 0,00000 & 0,00000 \\
\hline $\mathrm{px}_{1}$ & 0,00000 & 0,00000 & 0,01541 & 0,00000 & 0,00000 \\
\hline $\mathrm{px}_{2}$ & 0,00000 & 0,00000 & 0,09775 & 0,00000 & 0,00000 \\
\hline $\mathrm{px}_{3}$ & 0,00000 & 0,00000 & 0,31065 & 0,00000 & 0,00000 \\
\hline $\mathrm{px}_{4}$ & 0,00000 & 0,00000 & 0,49376 & 0,00000 & 0,00000 \\
\hline $\mathrm{px}_{5}$ & 0,00000 & 0,00000 & 0,33606 & 0,00000 & 0,00000 \\
\hline $\mathrm{py}_{1}$ & 0,00000 & 0,00000 & 0,00000 & 0,01541 & 0,00000 \\
\hline $\mathrm{py}_{2}$ & 0,00000 & 0,00000 & 0,00000 & 0,09775 & 0,00000 \\
\hline $\mathrm{py}_{3}$ & 0,00000 & 0,00000 & 0,00000 & 0,31065 & 0,00000 \\
\hline $\mathrm{py}_{4}$ & 0,00000 & 0,00000 & 0,00000 & 0,49376 & 0,00000 \\
\hline $\mathrm{py}_{5}$ & 0,00000 & 0,00000 & 0,00000 & 0,33606 & 0,00000 \\
\hline $\mathrm{pz}_{1}$ & 0,00000 & 0,00000 & 0,00000 & 0,00000 & 0,01541 \\
\hline $\mathrm{pz}_{2}$ & 0,00000 & 0,00000 & 0,00000 & 0,00000 & 0,09775 \\
\hline $\mathrm{pz}_{3}$ & 0,00000 & 0,00000 & 0,00000 & 0,00000 & 0,31065 \\
\hline $\mathrm{pz}_{4}$ & 0,00000 & 0,00000 & 0,00000 & 0,00000 & 0,49376 \\
\hline $\mathrm{pz}_{5}$ & 0,00000 & 0,00000 & 0,00000 & 0,00000 & 0,33606 \\
\hline
\end{tabular}

Pode-se observar que os conjuntos de base contraído e descontraídos foram representados por delimitadores diferentes. O conjunto de base descontraído é frequentemente representado entre parênteses, indicando em seu interior o número de primitivas de cada simetria, enquanto, o conjunto de base contraído é frequentemente representado entre colchetes e em seu interior indicase o número de agrupamentos dos diferentes tipos de simetria presente. É comum encontrar-se na literatura a identificação: conjunto de base (nsmp) contraída para [1skp], ou simplesmente $(\mathrm{nsmp}) \rightarrow$ [1skp]. A perda de energia para este exemplo específico é nula, uma vez que o conjunto de base contraído corresponde a solução das equações de Hartree-Fock. Entretanto, se esse mesmo conjunto de base contraído for transferido para uma molécula, tem-se diferenças significativas nas energias e outras propriedades em relação ao mesmo cálculo com o conjunto de base descontraído. Um exemplo pode ser visto na Tabela 6 em relação a cálculos envolvendo a molécula de $\mathrm{O}_{2}$. Uma maneira de minimizar-se as alterações nas propriedades em relação a contração do conjunto de base é evitar-se a contração das funções de base na região de valência. Bases nesta região devem ser mais flexíveis na descrição de propriedades eletrônicas. A Tabela 7 mostra que se o conjunto de base (9s5p) for contraído para [3s2p] obtém-se uma diferença em relação a energia total de apenas xxx u.a.

A simplicidade e facilidade de aplicação do método de contração de Raffenetti é contrabalanceada pela necessidade de repetir-se o mesmo conjunto de primitivas, tantas vezes quantos forem os orbitais atômicos do sistema 
em análise. Para conjuntos de base extensos a repetição do mesmo conjunto ou parte do conjunto que sofrerá contração aumentará significativamente, não o número final de integrais a serem armazenadas em disco, mas o número de integrais entre as primitivas. Uma alternativa utilizada em relação ao método de Raffenetti foi sugerida por Tom Dunning através de um método também denominado de método de contração segmentado [15].

Tabela 6: Cálculos para a molécula de $\mathrm{O}_{2}$ no estado fundamental $\left({ }^{3} \sum^{+} u\right)$ e geometria experimental ( $R e=2,280$ u.a.) em nível Hartree-Fock obtidos com: a) conjunto de bases gaussianas descontraído (9s5p), b) o mesmo conjunto contraído para $[2 \mathrm{~s} 1 \mathrm{p}] \rightarrow(711 / 41)$, c) conjunto de base (9s5p) contraído pelo método segmentado de Dunning para [3s2p] $\rightarrow$ (711/41) e conjunto de base de Dunning-Huzinaga (D95): [4s2p] $\rightarrow$ (6111/41)

\begin{tabular}{|l|l|l|l|}
\hline $\begin{array}{l}\text { Conjunto } \\
\text { de Base }\end{array}$ & Energia / u.a & $\begin{array}{l}\text { No. de Funções } \\
\text { de Base }\end{array}$ & $\begin{array}{l}\text { No. de } \\
\text { Primitivas }\end{array}$ \\
\hline$(9 \mathrm{~s} 5 \mathrm{p})$ & -149.514728502 & 48 & 48 \\
\hline$[2 \mathrm{~s} 1 \mathrm{p}]$ & -149.441888814 & 10 & 66 \\
\hline$[3 \mathrm{~s} 2 \mathrm{p}]$ & -149.148683319 & 18 & 50 \\
\hline$[4 \mathrm{~s} 2 \mathrm{p}]$ & -149.504762366 & 20 & 48 \\
\hline
\end{tabular}

A simplicidade e facilidade de aplicação do método de contração de Raffenetti é contrabalanceada pela necessidade de repetir-se o mesmo conjunto de primitivas, tantas vezes quantos forem os orbitais atômicos do sistema em análise. Para conjuntos de base extensos a repetição do mesmo conjunto ou parte do conjunto que sofrerá contração aumentará significativamente, não o número final de integrais a serem armazenadas em disco, mas o número de integrais entre as primitivas. Uma alternativa utilizada em relação ao método de Raffenetti foi sugerida por Tom Dunning através de um método também denominado de método de contração segmentado [15].

Este método também utiliza os coeficientes dos orbitais Hartree-Fock de cálculos atômicos como coeficientes de contração das bases. Entretanto, as funções primitivas raramente são repetidas em diferentes grupos de funções contraídas e os coeficientes de contração são selecionados seletivamente em função da maior participação da primitiva em determinado orbital atômico. Para exemplificar, basta observar os coeficientes de combinação linear apresentados na Tabela 5 para o átomo de oxigênio obtidos através de um cálculo Hartree-Fock com o conjunto de coeficientes de contração fornecidos por Dunning na Tabela 7 para o mesmo átomo. Esta comparação mostra que Dunning sugere que os coeficientes de contração das funções com expoentes maiores (mais próximas da região nuclear) sejam provenientes do orbital 1s, que descreve a distribuição eletrônica na região interna. $\mathrm{O}$ número de funções que serão selecionadas para este primeiro grupo se estende até a região do orbital 1s em que os coeficientes de combinação linear apresentam um valor significativo. Para o segundo grupo, que provavelmente corresponde ao de funções mais próximas da região de valência, Dunning sugere que os coeficientes sejam obtidos através das contribuições mais significativas do orbital 2s. Pode-se perceber que a função com expoente $\alpha=9,5320$ está sendo repetida no segundo grupo de funções contraídas. Observando-se os coeficientes de combinação linear no cálculo HartreeFock, verifica-se que esta função apresenta um valor significativo, tanto no orbital atômico 1 s, quanto 2 s, e que portanto, deve ser importante na descrição da distribuição eletrônica desses dois orbitais. Os coeficientes de contração desse segundo grupo de funções diferem dos coeficientes dos orbitais Hartree-Fock porque foram renormalizados. Dunning sugere que as primitivas que descrevem a região mais externa ( expoentes menores ) devam permanecer descontraídas e portanto, apresentam coeficientes de contração iguais a 1 , o que indica que não estão sendo agrupadas com nenhuma outra função. Para as funções $\mathrm{p}$ as mesmas regras se aplicam. Consequentemente, o conjunto de base apresentado na Tabela 7 corresponde a uma contração [3s2p], uma vez que foram criados 3 grupos de funções s e dois grupos de funções p.

Tabela 7: Conjunto de base (9s5p) contraído pelo método segmentado de Dunning para [3s2p].

\begin{tabular}{|l|l|l|l|}
\hline $\boldsymbol{\alpha}_{\mathbf{S}}$ & {$[\mathbf{3 s}]$} & $\boldsymbol{\alpha}_{\mathrm{p}}$ & {$[\mathbf{2 p ]}$.} \\
\hline 7817,0000 & 0,001176 & 35,1800 & 0,019580 \\
\hline 1176,0000 & 0,008968 & 7,9040 & 0,124200 \\
\hline 273,2000 & 0,042868 & 2,3050 & 0,394714 \\
\hline 81,1700 & 0,143930 & 0,7171 & 0,627376 \\
\hline 27,1800 & 0,355630 & 0,2137 & 1,000000 \\
\hline 9,5320 & 0,461248 & & \\
\hline 3,4140 & 0,140206 & & \\
\hline 9,5320 & $-0,154153$ & & \\
\hline 0,9398 & 1,056914 & & \\
\hline 0,2846 & 1,000000 & & \\
\hline
\end{tabular}

Pode-se perguntar por que, neste exemplo, o conjunto (9s5p) foi contraído para [3s2p] e não [4s3p] ou um outro conjunto qualquer? Como mencionado anteriormente, a idéia básica é minimizar-se o número de funções de base através da formação de grupos e quanto menor o grupo, menor o número de integrais a serem armazenadas em disco. O conjunto [3s2p], segundo Dunning, para este caso é o menor conjunto que pode ser formado produzindo diferenças aceitáveis nos valores de propriedades moleculares em relação aos cálculos com o conjunto de base descontraído.

Deve-se chamar a atenção para o fato de que Dunning, não definiu os conjuntos de contração em relação às 
diferenças entre cálculos atômicos com o conjunto de base descontraído e contraído, mas sim, selecionando moléculas e avaliando se o conjunto contraído não produzia, em ambiente molecular, distorções significativas em relação aos cálculos com o conjunto de base descontraído. Desta forma, embora a perda de qualidade de propriedades em ambiente atômico pudesse ser significativa, em ambiente molecular as diferenças deveriam ser aceitáveis.

O grande inconveniente do esquema de Dunning está na escolha arbitrária dos coeficientes de contração entre os diferentes orbitais atômicos ocupados. Para os átomos mais leves da tabela periódica este não é um problema sério, porém para elementos mais pesados onde vários orbitais atômicos são possíveis, a seleção dos conjuntos a serem formados é um trabalho extremamente difícil de ser realizado.

\section{O método de orbitais naturais}

Uma vez que o desempenho dos computadores modernos tem crescido significativamente, tem sido uma tendência realizar-se cálculos incluindo efeitos de correlação eletrônica, ou seja, corrigindo a deficiência introduzida pelo método de Hartree-Fock ao assumir a aproximação do campo médio. Uma das maneiras de corrigir-se os efeitos de correlação eletrônica é através da mistura de determinantes de Slater de diferentes configurações eletrônicas. Esta mistura de configurações permite a obtenção de um termo denominado de matriz densidade de primeira ordem que pode ser convenientemente manipulado para produzir coeficientes de combinação linear das funções de base, não em termos de um único determinante de Slater, mas em termos da mistura de diversas configurações eletrônicas. Estes coeficientes de combinação linear resgatam a lembrança nesses cálculos multiconfiguracionais da imagem dos orbitais de HartreeFock e portanto são denominados de orbitais naturais [17].

Considerando-se esta informação pode-se utilizar os coeficientes provenientes de orbitais naturais para realizar contrações de conjuntos de base. Normalmente, o uso de orbitais naturais tem sido acompanhado do esquema de contração de Raffenetti. Neste caso, o número de conjuntos contraídos pode ser definido em termos de uma medida do número de ocupação eletrônico em cada orbital natural. A escolha do limite para esta população é arbitrária e o controle do tamanho do conjunto de base contraído é determinado em função da proximidade dos resultados calculados com o conjunto de base contraído e descontraído.

\section{Outros aspectos}

A possibilidade de agrupar-se primitivas através de métodos de contração geraram diferentes formas de representação dos conjuntos contraídos. Em capítulo anterior mencionou-se a representação $(\mathrm{nsmp}) \rightarrow$ [1skp]. Entretanto, a representação [1skp] não torna explícito o número de primitivas em cada grupo. Uma alternativa sugerida por Pople foi a de representar os diferentes grupos de funções pelo número de primitivas. Desta forma, segundo a notação sugerida por Pople, um conjunto contraído 3-21G seria a representação de um conjunto de base descontraído (6s3p) onde 3 primitivas formariam um primeiro grupo, 2 primitivas um segundo grupo e 1 primitiva um terceiro grupo. O hífen indica uma separação entre a região de valência da região mais interna. Segundo Pople, os grupos a esquerda do hífen representariam aqueles pertencentes aos orbitais próximos dos núcleos, enquanto que os grupos a direita do hífen a região de valência. A letra $G$ colocada no final da representação da base contraída indica que esta é uma contração de funções gaussianas. A estrutura eletrônica do átomo de $\mathrm{C}$ no estado fundamental apresenta a configuração: $1 \mathrm{~s}^{2}$ $2 \mathrm{~s}^{2} 2 \mathrm{p}^{2}$. Desta forma, um conjunto de base contraído 3$21 \mathrm{G}$ apresentará um grupo de 3 funções s representando a região mais próxima do núcleo e na região de valência um grupo de funções possuindo duas funções s e um grupo de funções possuindo apenas uma função do tipo s. Uma vez que a estrutura eletrônica do átomo possui funções $p$ na região de valência, a representação 21 indica também que há um grupo de funções de valência contendo duas funções $\mathrm{p}$ e um grupo de funções possuindo apenas uma função do tipo p. Em outras palavras, a representação da região de valência 21 indica o número de grupos de primitivas e quantas primitivas existem em cada grupo. A notação deixa implícito o tipo de funções de base em função da configuração eletrônica do átomo em questão.

Para átomo mais pesados uma base $3-21 \mathrm{G}$ indica que cada nível de energia interno é representado por grupos de funções contendo 3 primitivas cada e na região de valência dois grupos com 2 primitivas e 1 primitiva respectivamente. O átomo de cloro com uma distribuição eletrônica $1 s^{2} 2 s^{2} 2 p^{6} 3 s^{2} 3 p^{5}$, quando representado por um conjunto de base $3-21 \mathrm{G}$ deve indicar que na região interna os orbitais atômicos 1 s 2 s 2 p estarão sendo representados por conjuntos de 3 primitivas cada e na região de valência (3s3p) por dois conjuntos contendo 2 e 1 funções em cada grupo.

Pople além de sugerir outros conjuntos de base, tais como: 4-31G, 6-31G, 6-311G, também sugeriu uma representação para a inclusão de funções de polarização e de difusas. Como em cálculos convencionais o número 
de funções de polarização e de difusas não ultrapassa a uma primitiva de cada tipo, Pople sugeriu que funções de base contendo funções de polarização deveriam ser representadas por $\left({ }^{*}\right.$ ou **) e funções difusas por $(+$ ou ++$)$. Um único* ou + indicaria a inclusão das respectivas funções em todos os átomos diferentes do hidrogênio e um par de ** ou ++ indicaria a inclusão de funções de polarização e difusas em todos os átomos. Desta forma, conjuntos de base $6-31 \mathrm{G}^{*}$ estariam indicando a inclusão de uma função de polarização sobre todos os átomos diferentes do hidrogênio. Um conjunto de base $6-311+G^{* *}$ indicaria a inclusão de funções difusas em todos os átomos diferentes do hidrogênio e a inclusão de funções de polarização em todos os átomos do sistema.

Um outro conjunto de base contraído bastante divulgado na literatura corresponde aos conjunto STO-nG, onde $\mathrm{n}=2,3, \ldots$ Estes conjuntos de base correspondem a tentativa de reproduzir o comportamento de funções de Slater através da combinação de funções gaussianas [18]. O número $\mathrm{n}$ indica o número de funções gaussianas necessárias para representar uma função de Slater. A obtenção dos coeficientes de mistura da funções gaussianas neste caso não é feita no sentido variacional, mas sim procurando minimizar o desvio entre a função de Slater em questão e o conjunto de funções gaussianas que procurarão reproduzir as suas características.

\section{Considerações finais}

A escolha do conjunto de base para um cálculo qualquer não é trivial. Envolve não somente a melhor representação possível da distribuição eletrônica de um sistema qualquer, mas também os recursos computacionais disponíveis. $\mathrm{O}$ compromisso entre estes dois aspectos normalmente norteiam a escolha. Considerando-se uma situação computacionalmente ideal, pode-se dizer que os conjuntos de base maiores e contendo a maior diversidade de tipos de funções de base $(s, p, d, \ldots)$ tendem a representar de maneira mais adequada a distribuição eletrônica. $\mathrm{Na}$ prática, observa-se que, em alguns casos, o uso de bases muito estendidas tendem a produzir resultados em nível Hartree-Fock que diferem mais significativamente de dados experimentais do que aqueles produzidos por bases mais modestas. Em princípio, os cálculos com bases estendidas são mais corretos no sentido de que devem se aproximar ao que se chama de um conjunto de base completo para caracterizar a nuvem eletrônica do sistema. As diferenças entre os valores calculados com a base estendida e os dados experimentais mostram simplesmente que a aproximação de Hartree-Fock não está sendo adequada para produzir as propriedades de interesse. Neste caso, a única coisa alternativa a ser tentada será o aperfeiçoamento do método Hartree-Fock através da correção dos efeitos de correlação eletrônica. De maneira pictórica, pode-se dizer que o método Hartree-Fock sofre de um problema de alto grau de "miopia" eletrônica. Ele pode perceber a presença dos elétrons ao redor dos núcleos, mas não consegue focá-los adequadamente. Mesmo com o melhor conjunto de base existente, esse tipo de problema persistirá. Para algumas propriedades moleculares, esta miopia eletrônica podem não ser importantes, mas para outras é crucial. Desta forma, se empregando-se o melhor conjunto de base existente, não se consegue os resultados mais adequados em nível Hartree-Fock, deve-se melhorar os efeitos de correlação eletrônica. O melhor desempenho de conjuntos de bases menores para o cálculo de algumas propriedades em determinada circunstância são reflexo de um cancelamento de erros apropriado. Mas, não garantem que a aplicação desse mesmo conjunto de base para o cálculo de outra propriedade irá produzir resultados tão adequados. Como lembrete vale destacar que deve-se empregar métodos de correlação eletrônica quando se possui um bom cálculo Hartree-Fock, e este é obtido a partir de um conjunto de funções de base adequado.

Referências Bibliográficas

1. Hartree, D.R., "The Calculation of Atomic Structures ", John Wiley \& Sons, New York (1957).

2. Roothaan, C.C.J., Rev.Mod.Phys. 1951, 23: 69.

3. Davidson, E.R.; Feller, D., Chem.Rev. 1986, 86: 681. b) Feller, D.; Davidson, E.R., 'Reviews in Computational Chemistry", Editors: K.B.Lipkowtiz \& D.B.Boyd, VCH, New York (1990). c) Shavitt, I., Israel J. Chem. 1993, 33: 357. d) Andzelm, J.; Klobukowski, M.; Radzio-Andzelm, E.; Saski, Y.; Tatewaki, H., 'Gaussian Basis Set for Molecular Calculations”, Editor: S. Huzinaga, Elsevier, Amsterdan, (1984).

4. Shavitt, I., 'Methods in Computational Physics", vol.2, Academic Press, New York (1963).

5. Epstein, S., "The Variational Method", New York (1974).

6. Bardo, R.D.; Ruedemberg, K., J.Chem.Phys. 1974, 
60: 918.

7. Huzinaga, H.; Klobukowski, M.; Tatewaki, H., Can.J.Chem. 1985, 63: 1812.

8. Silver, D.M.; Wilson, S.; Nieuwpoort, W.C., Int. J.Quantum Chem. 1978, 14: 635. b) Custodio, R.; Goddard, J.D.; Giordan, M.; Morgon, N.H., Can. J.Chem. 1992, 70: 580. c) Malli, G.L.; da Silva, A.B.F. e Ishikawa, Y., Phys.Rev. 1993, 47: 143.

9. Deb, B.D., 'The Force Concept in Chemistry", Van Nostrand, New York (1981).

10. Burden, F.R.; Wilson, R.M., Advan.Phys. 1972, 21: 825 .

11. Nakatsuji, H.; Kanda, K.; Yonezawa, T., Chem. Phys.Lett. 1980, 75: 340. b) Nakatsuji, H.; Kanda, K.; Hada, M.; Yonezawa, T., J.Chem.Phys. 1982, 77: 3109 .

12. Custodio, R.; Goddard, J.D., J.Mol. Struct.(Theochem) 1992, 277: 263.

13. Vianna, R.O.; Custodio, R.; Chacham, H.; Mohallem, J.R., Int.J.Quantum Chem. 1992, 26: 311.

14. Custodio, R.; Goddard, J.D., J.Mol. Struct.(Theochem) 1993, 281: 75.

15. Dunning, T.H.; Hay, P.J., "Theoretical Chemistry", editor: H.F.Schaefer III, Plenum Press, New York, cap.1 (1976), p.1-28.

16. Raffenetti, R.C., J.Chem.Phys. 1973, 58: 4452.

17. Almlof,J.; Helgaker, T.; Taylor, P.R., J.Phys.Chem. 1988, 92: 3029. b) Almlof, J. e Taylor, P.R., Adv. in Quant. Chem. 1991, 22: 301.

18. O-ohata, K.; Taketa, H.; Huzinaga, S., J.Phys.Soc. Japan 1966, 21: 2306. 